\title{
Providers as Stakeholders in Addressing Implementation Barriers to Youth Mental Healthcare
}

\author{
Eric T. Goodcase ${ }^{1}\left[\right.$ ] Alexis M. Brewe ${ }^{2} \cdot$ Susan W. White ${ }^{2} \cdot$ Shane Jones $^{2}$ \\ Received: 28 March 2021 / Accepted: 7 October 2021 / Published online: 20 October 2021 \\ (c) The Author(s), under exclusive licence to Springer Science+Business Media, LLC, part of Springer Nature 2021
}

\begin{abstract}
Research has shown that youth with mental health disorders often do not receive adequate care. School- and communitybased mental health organizations are integral to implementing evidence-based mental healthcare to the vast majority of youth. It is therefore important to understand the perspectives of this stakeholder group, to determine how to improve access to high-quality care. A series of three focus groups with community mental health providers and three school counselors and social workers focus groups were conducted to get their perspective on existing barriers that prevent youth who need mental health services from being treated. A grounded theory inductive qualitative analysis revealed six major themes (Lack of Services, Lack of Knowledge, Stigma, Logistics, Poor Past Experiences with Mental Health, and Poor Coordination of Services). Each of these themes are discussed and implications are framed within the context of implementation science.
\end{abstract}

Keywords Implementation $\cdot$ Youth $\cdot$ Mental health $\cdot$ School-based $\cdot$ Community

\section{Introduction}

Most mental health disorders emerge in late childhood and early adolescence (Das et al., 2016), although symptoms can occur much earlier. In fact, one in five children and adolescents need mental health services, yet most do not receive any services (Green et al., 2013; Merikangas et al., 2011). Scientific evidence indicates that many disorders and problem behaviors (e.g., suicidal ideation, violence, depression) in youth are treatable, and sometimes preventable, through evidence-based interventions (EBIs), including targeted prevention and intervention programs. Unfortunately, there is a translation gap in bringing research-supported, effective interventions into practice in communities and schools. Implementation science, or the scientific study of methods to increase the use of evidence-based innovations into routine settings (Eccles

Eric T. Goodcase

etgoodcase@ches.ua.edu

1 Department of Human Development and Family Studies, University of Alabama, 214 Child Development Research Center, Tuscaloosa, AL 35487, USA

2 Center of Youth Development and Intervention, University of Alabama, 200 Hackberry Lane, Tuscaloosa, AL 35487, USA
\& Mittman, 2006), seeks to close this uptake gap. Arguably, the most widely used implementation science framework is the Consolidated Framework for Implementation Research (CFIR; Damschroder et al., 2009), developed to guide systematic assessment of multilevel implementation contexts to identify the factors that influence intervention implementation and effectiveness (Kirk et al., 2016).

Results from one of the largest longitudinal studies of mental health and service use indicated that the education sector is the most common point of entry into services for youth (Farmer et al., 2003). Additionally, children are more likely to receive services at school than through any other service sector including mental health clinics, medical facilities, and the child welfare system, particularly if they are on public insurance, are from low-income households, and/or are racial and ethnic minorities (Ali et al., 2018). However, though children and adolescents from low income households have higher needs, schools in low income areas may struggle relative to other schools to keep up with these needs, implement mental health interventions, and to get faculty and staff engaged with interventions (Cappella, et al., 2008), Mental health services delivered in most schools are not consistently evidencebased, rigorously implemented, or sustained over time (Ali et al., 2018; Gottfredson \& Gottfredson, 2002), which may result in suboptimal outcomes for the child and wasted 
resources (Ali et al., 2018). Since schools are an important access point for children to receive services, it is important to understand what is occurring in this environment that promotes or hinders a child's access to quality mental health services.

\section{Barriers to Treatment}

The vast majority of the literature on barriers for youth accessing mental health services focuses on the direct personal experiences of youth and their caregivers. One such meta-analysis (Reardon et al., 2017) of existing studies utilizing self- and caregiver-report found four overall themes related to perceived barriers to youth accessing mental health services. These barriers were (1) systematic and structural issues (e.g., transportation, cost of services and lack of insurance, lack of service providers), (2) views and attitudes towards treatment (e.g., consequences of child having a diagnosis, trust in service providers, and social stigma about mental health), (3) knowledge of mental health and the help-seeking process (e.g., parent's recognition of the problem and its severity, knowledge of appropriate providers), and (4) specific family circumstances (e.g., limited ability to commit time needed for treatment and lack of family support). These themes are similar to other literature on barriers to treatment (Corrigan et al., 2014; Dempster et al., 2013; Gulliver et al., 2010), although research suggests that families in more rural communities may experience additional challenges in accessing services due to a lack of mental health providers, fewer financial resources to access those limited providers, lower mental health literacy, and increased social stigma related to mental illness (GirioHerrera et al., 2013; Pullmann et al., 2010; Repie, 2005; Weist et al., 2000).

Consistent with the CFIR model, which stresses the importance of understanding the perspectives of all stakeholders involved in the mental health care process to determine factors that stymy or facilitate implementation (Damschroder et al., 2009), it is important to consider the perspectives of stakeholders who provide services. Community mental health providers (CMHP) who work with children are involved in the process of referring, diagnosing, and treating youth mental health problems. A few previous studies have assessed the perceptions of CMHP and have identified cultural factors (e.g., stigma and perceptions of CMHP), client level factors (e.g., not viewing treatment as relevant or lack of funds), provider factors (e.g., high caseload), and process related factors (e.g., poor therapist-client fit) as things that influence the likelihood for individuals initiating and consistently attending therapy (Jensen et al., 2020; Kim \& Salyers, 2008; Stevens et al., 2006). However, there are few studies like this that consider provider perspectives of barriers related to youth attending therapy and many of those that do exist often are unique to particular treatments or contexts (e.g., telehealth or rural environments).

School counselors and social workers (SC/SW) are often tasked with working with students and school personnel to improve the school environment. SC/SWs are in a unique position to both identify youth who are at-risk and in need of referral, and to be the liaison between families and other service providers (Baggish \& Hardcastle, 2005; Girio-Herrera et al., 2013; Splett et al., 2013). Thus, their perspectives could provide valuable information on the barriers to youth mental health care for families who ultimately seek services for their children and, possibly more importantly, the factors that prevent some families from seeking services. Repie (2005) found that in-school stakeholders (i.e., school counselors, school psychologists, and teachers) believed that that the level of stress in the family unit prior to seeking services, financial problems, and social stigma about mental health problems were common barriers.

\section{Current Study}

The intention of the current study is to better understand the barriers, as perceived by providers, that impede the recognition of youth mental health problems, children's access to mental health services, and successful treatment. While previous studies have explored this concept, many of these studies focused on parents, families, or children/adolescents themselves (Reardon et al., 2017). Prior work has explored this in relation to school-based mental health (e.g., Repie, 2005) and CMHP perceptions (Jensen et al., 2020; Kim \& Salyers, 2008; Stevens et al., 2006). The current study sought to contribute to our extant knowledge of barriers and facilitators of implementation of EBI for youth mental health, via a qualitative study of provider perspectives. We include SC/SW and CMHP because of the probability that they share similar experiences with respect to implementation; however, we also consider points where perspectives may differ.

\section{Method}

\section{Procedure}

Focus groups were used to gather in-depth data on the attitudes, feelings, and beliefs related to implementation of mental healthcare from a purposely selected group of providers in a relatively brief period of time. Qualitative data from semi-structured focus groups were analyzed utilizing an inductive grounded theory approach (Corbin \& Strauss, 2008) to examine provider perspectives. A total of 6 focus 
groups were conducted, 3 with CMHPs and 3 with SC/SWs. CMHPs were recruited through contacting different agencies in the area and providing them a Qualtrics link with information about the study, informed consent information, and an opportunity to sign up for a particular date. SC/SW were recruited through the district's assessment coordinator. SC/ SW were instructed to review the online sign up and were notified that their participation was voluntary. Focus groups were conducted by one of three authors and trained via the first author to maintain consistency across groups. A note taker, either one of the authors or an undergraduate research assistant trained by one of the authors, was present to keep track of who was speaking and what was said. After signing a consent form, participants filled out a demographic survey before the focus group began. Focus groups followed a semi-structured interview protocol and lasted approximately 60-90 min long.

Focus groups were semi-structured in order to maintain consistency in information across all focus groups but still allowed for flexibility within the unique groups. The protocol was made up of four questions asking participants what they perceive to be the current state of mental health for local youth. Specifically, they were asked what were the most common mental health concerns they see; what the typical process is to secure treatment for youth from recognition of a problem to receival of services; what barriers exist that inhibit treatment; and what the communication looks like between schools, parents, and CMHPs. A full copy of the focus group protocol can be viewed as supplemental materials.

\section{Participants}

Both CMHP and SC/SW for this study were recruited from a city in the Southeastern United States. Based on population, the city would qualify as an urbanized area based on definitions from the Census Bureau (census.gov). The most prominent racial group in the city is white $(>50 \%)$ followed by black ( $>40 \%$ ), with minimal numbers of other racial groups. The city's median income is significantly lower than U.S. median but only slightly lower than the state median income. The school district from which the SC/SWs were recruited is made up of 21 schools and 11,000 students. The majority of students in this district are black ( $>70 \%)$ and qualify for free and reduced lunch ( $>60 \%)$.

School Counselors and Social Workers. The three SC/ SW focus groups made up a total of 35 participants (34 females). School counselors made up $65.7 \%$ of the sample $(n=23), 31.4 \%$ were school social workers $(n=11)$, one participant was an administrator, and one did not answer. In regards to race and ethnicity, $40 \%$ were white $(n=14)$, $57.1 \%$ were black $(n=20)$, and one participant did not answer. Participants were an average of 40.94 years old (Range: $24-60, S D=7.65$ ) and had an average of 9.61 years of experience in their position (Range: $0.5-31, S D=7.11$ ).

Community Mental Health Providers. The three CMHP focus groups were made up of a total of 13 participants (11 female). Participation was open to any CMHPs (i.e., Licensed Professional Counselors, Marriage and Family Therapists, Psychologists, \& Psychiatrists) who provided direct mental health treatment to children and/or provided supervision to youth CMHPs. Participants were mostly white (92.3\%) with one participant who identified as multiracial. In terms of education and field, eight of the participants had a Master's degree (six in Marriage and Family Therapy and 2 in Social Work), 4 had a PhD (two in Psychology, one in Social Work, and one in Marriage and Family Therapy), and one had an MD (Child Psychiatry). Participants were an average of 40.67 years old (Range: $25-69, S D=14.7$ ) and had an average of 10.71 years of experience (Range: 9 months -30 years, $S D=8.44$ ).

\section{Analytic Approach}

Transcripts from each focus group were transcribed into a word processor and were imported into QDA Miner Lite software (QDA Miner, 2016). A group made up of the lead researcher and three other members of the research team engaged in qualitative analysis. Each member of the research team reviewed the transcripts on their own to get familiar with the data before convening as a group and reviewing the transcripts line by line and engaging in open coding (Corbin \& Strauss, 2008). The group discussed emerging themes related to barriers that exist for children needing mental health services, defined them, and differentiated or synthesized them based on those definitions. Differences in opinions were openly discussed until the research team reached consensus. Axial coding (Corbin \& Strauss, 2008) was accomplished through group discussion to categorize similar codes into larger themes based on similarities between the open codes (e.g., School Personnel Lack of Knowledge and Parents Lack of Knowledge being grouped into a larger category of Lack of Knowledge about Mental Health).

In conducting a more inductive approach to analysis, it is important to acknowledge the positionality of the authors. Each of the authors and coders, with the exception of the undergraduate research assistant, are individuals with higher degrees in a mental health related field (Marriage and Family Therapy, Clinical Psychology, and Social Work). This training may make each of the authors aware of the significance of the problem being studied and help them relate to the CMHP participants in the study. However, even though each of the authors has some experience working with schools 
and/or providing mental health treatment in a school system they may have different perspectives than SC/SW and this may influence how focus groups were conducted and how results were interpreted.

\section{Results}

Grounded theory driven inductive analysis from the six focus groups produced six distinct categories of themes about the barriers to children receiving mental health services: (a) Lack of Available Resources, (b) Lack of Knowledge Around Mental Health, (c) Mental Health Stigma, (d) Issues with Logistics, (e) Poor Experiences with Mental Health, and (f) Poor Coordination of Services. Each of these categories had a number of subthemes that were mentioned by CMHPs, SC/SWs, or both. Themes, subthemes, quotations, and the frequency of each subtheme are available in Table 1.

\section{Lack of Available Resources}

Both CMHPs and SC/SWs discussed that limited availability of resources in the community was a significant block to children and adolescents not receiving appropriate services. The lack of CMHPs in the community was identified as an issue by all CMHP groups and two SC/SW groups. All CMHP groups and one SC/SW group mentioned that there is a shortage in specialists such as child psychiatrists, clinicians that specialize in working with children or specialize in play therapy, or clinicians that specialize in treating particular diagnoses like Autism Spectrum Disorder. Additionally, both CMHP and SC/SW discussed the importance of having providers who can speak different languages and/ or competently treat individuals from other cultures. The CMHP group also indicated that this lack of providers results in caseloads that are too large or therapists who are overworked, as well as delays in treatment due to long waitlists. Additionally, both groups mentioned a need for local crisis or emergency services.

Unique to the school environment, all $3 \mathrm{SC} / \mathrm{SW}$ groups and one CMHP group identified that the ratio of students to $\mathrm{SC} / \mathrm{SWs}$ is too large. This ratio makes it more difficult for SC/SWs to intervene with students or establish other programs or interventions that could benefit students. This difficulty is amplified by school counselors having too many different duties and not being able to focus on interventions that they believe could be helpful long term.

\section{Lack of Knowledge Around Mental Health}

Another important barrier for children and adolescents receiving services is that youth and the important adults in their life may not have adequate knowledge about mental health and which behaviors may indicate a problem, or there are other issues in the environment that prevent adults noticing or acting on potential issues. Every focus group identified parents' lack of knowledge or familiarity with mental health as a key reason why youth often do not receive adequate mental health care. Often times, parents do not seek mental health treatment until it is necessary to do so because of an emergency or because the problem is disruptive enough that it is affecting the parents at home. Both CMHPs and SC/SWs noted that there are additional family system issues associated to parents' lack of knowledge that lead them to not being as committed as necessary for quality care, such as a hesitancy to involve the family unit in therapy for their child or the parents needing their own mental health treatment themselves. Parent defensiveness was identified as an associated barrier to quality mental health treatment for their children as they may think this reflects on them negatively.

Both CMHP and SC/SW noted that even if parents recognize a mental health problem exists, they may lack the knowledge about how or where to seek services for the problem. Without a clear path forward to mental health services, parents often do not know how to begin to access the care that their child needs. Due to parents' lack of knowledge of both mental health problems and the pathway to treatment, both groups discussed that parents often opt to seek out a medical option (i.e., medical doctors and pediatricians) first. Both CMHPs and SC/SWs also identified that, in their experiences, parents sometimes have a desire for a 'quick fix' such as psychotropic drugs rather than being willing to pursue a more long-term therapeutic option for their child.

Given that children spend a significant portion of their days at school, both CMHPs and SC/SWs agreed that teachers' and school personnel's limited knowledge on mental health resources also serves as a barrier to children getting adequate mental health services because it delays identification of the problem. Recognizing a student's symptoms becomes even more difficult when that student's academic performance may mask those symptoms for teachers. One SC/SW focus group identified that other external school barriers, such as teachers having many students or only seeing them for one period of the day (i.e., middle and high school teachers) can prevent teachers from identifying mental health symptoms in their students. One CMHP and two SC/ SW focus groups identified that school counselors not being trained to do long-term effective therapy is a particular barrier for students in need of treatment. 


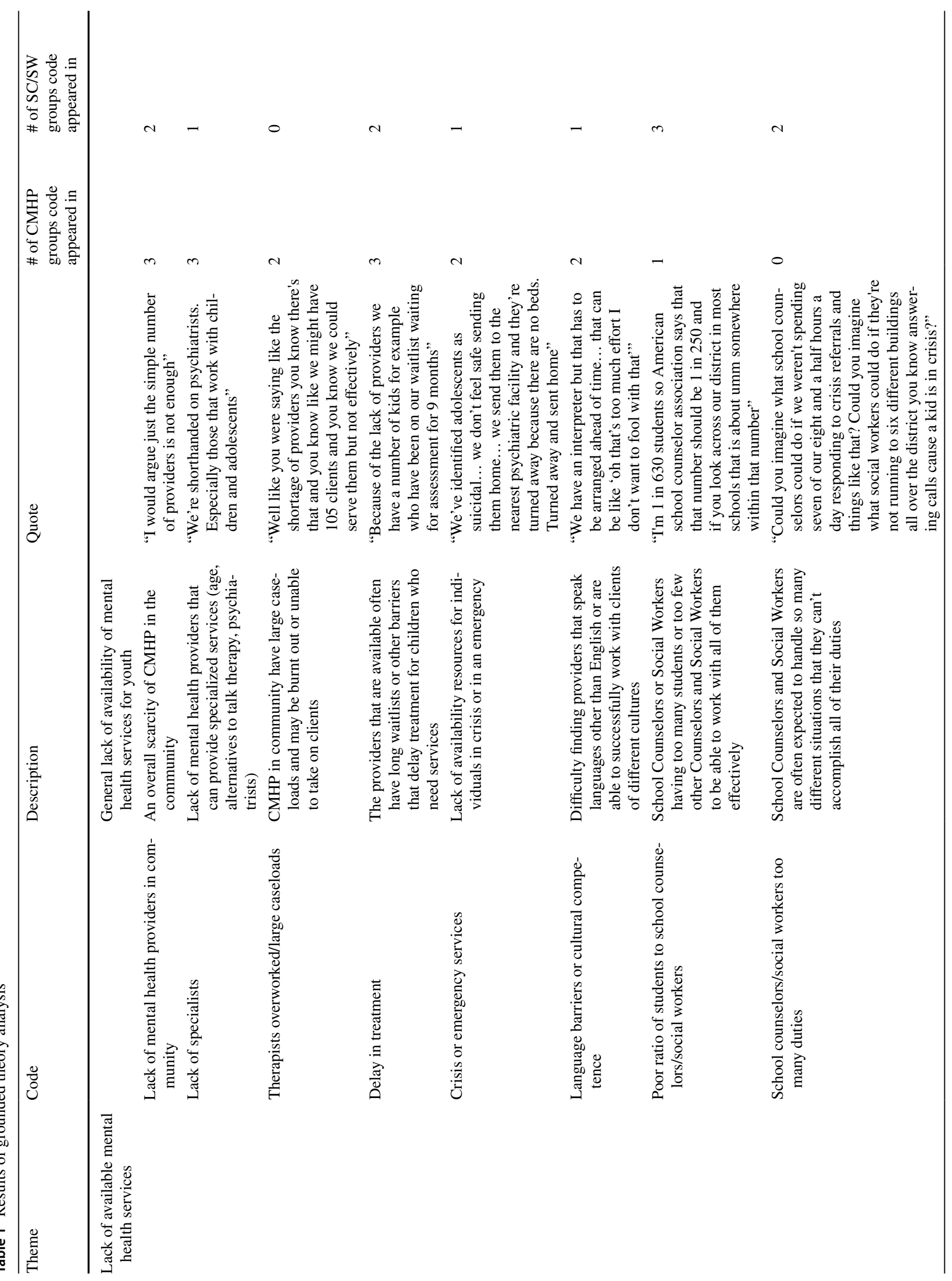




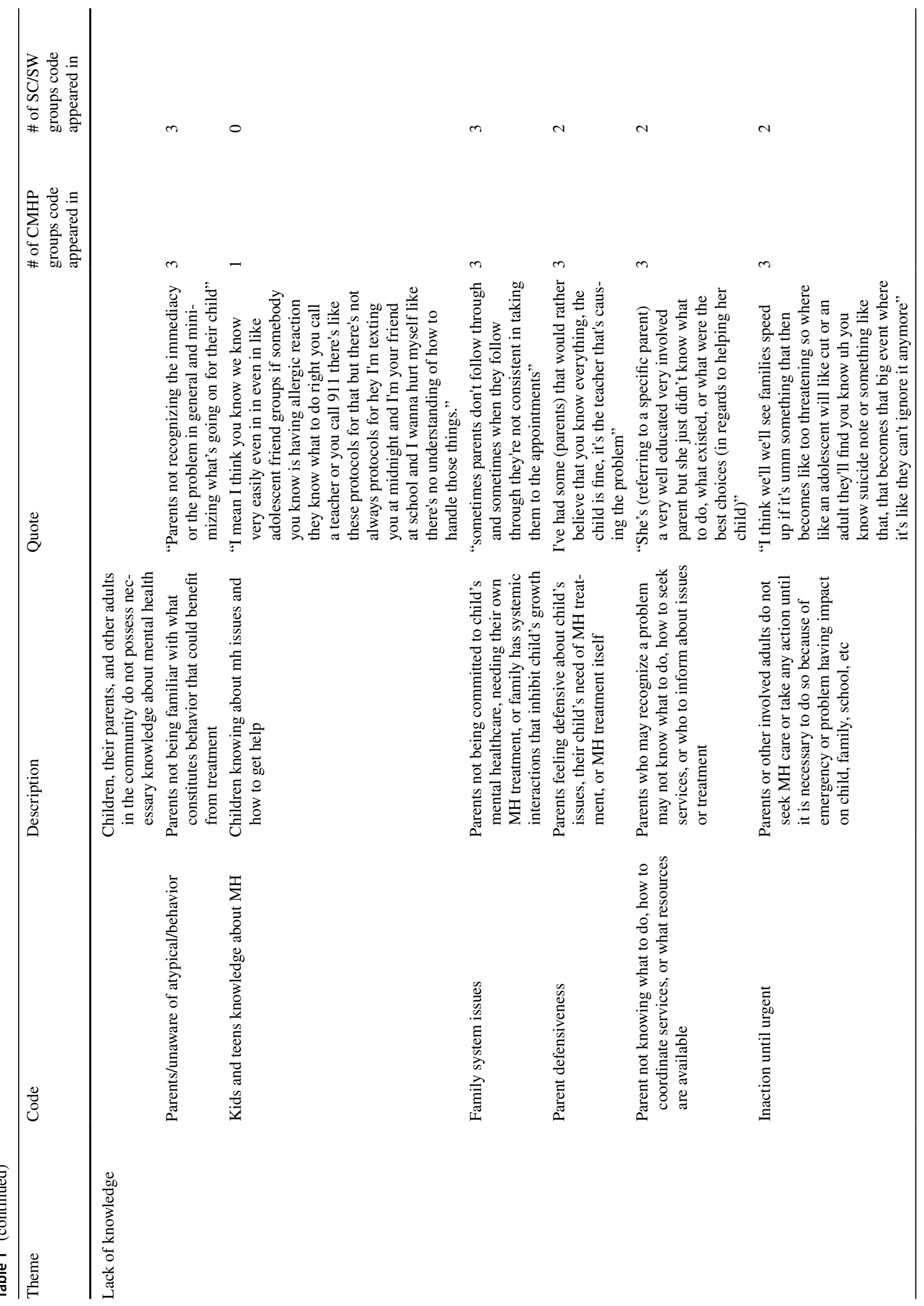




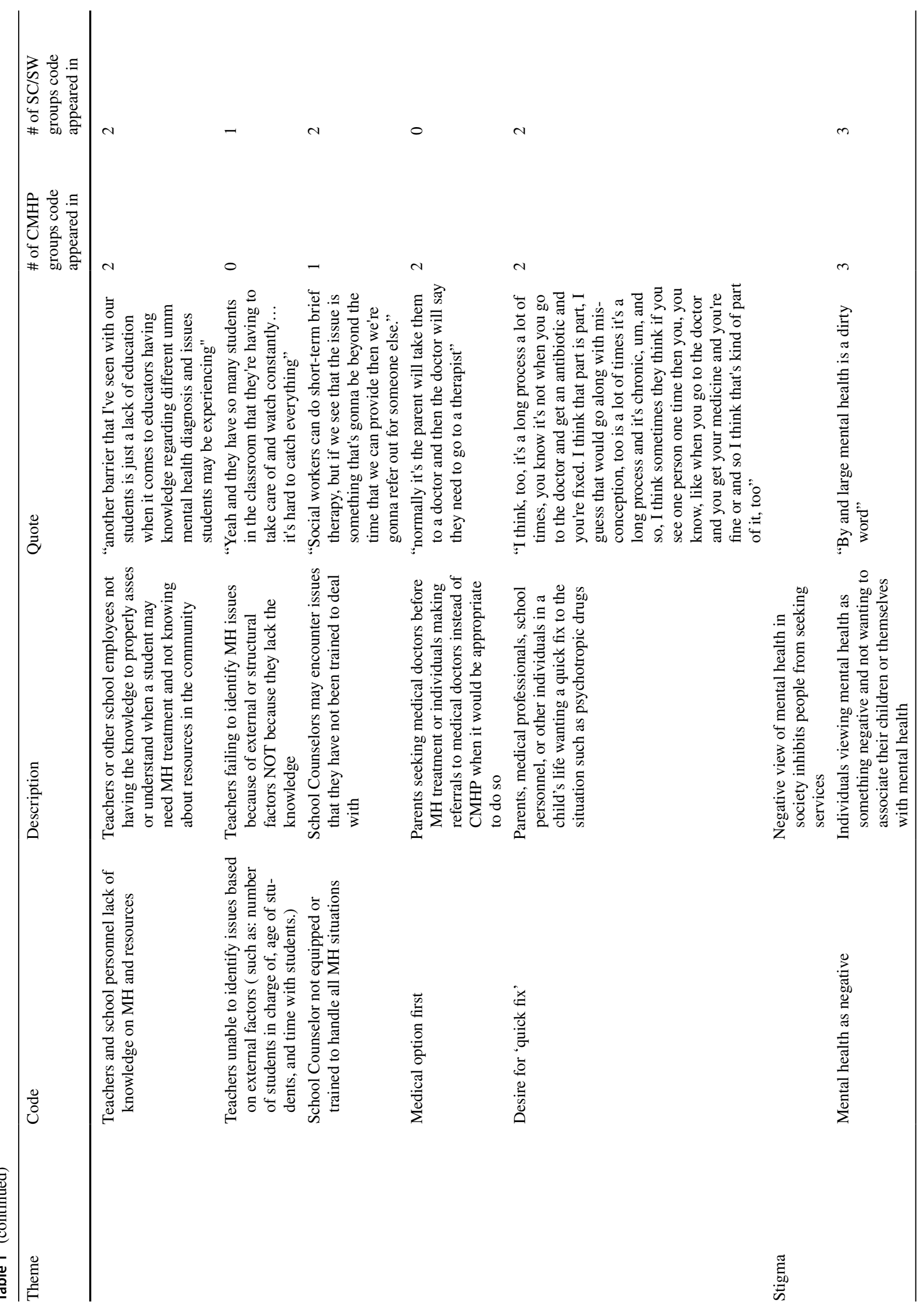




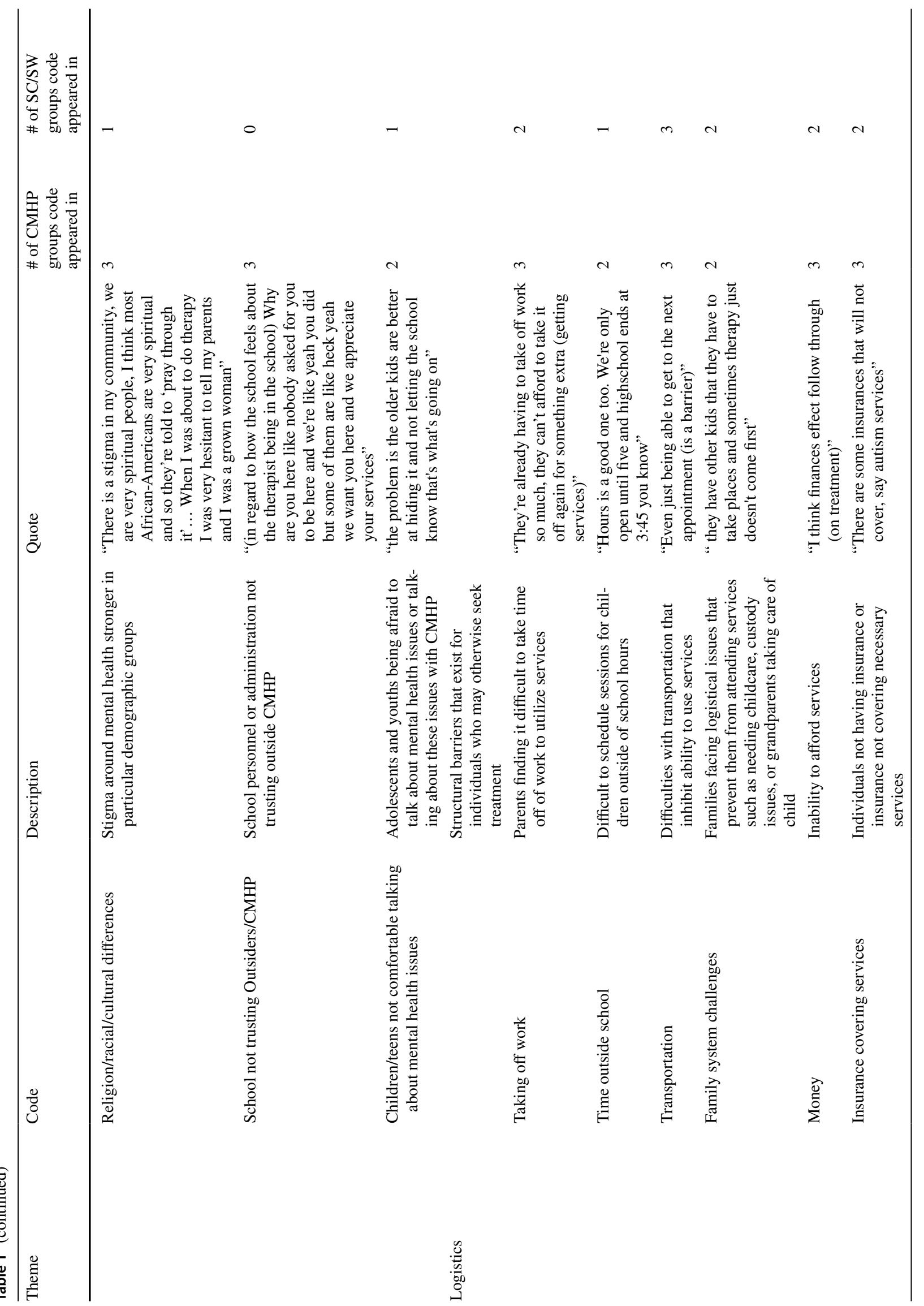




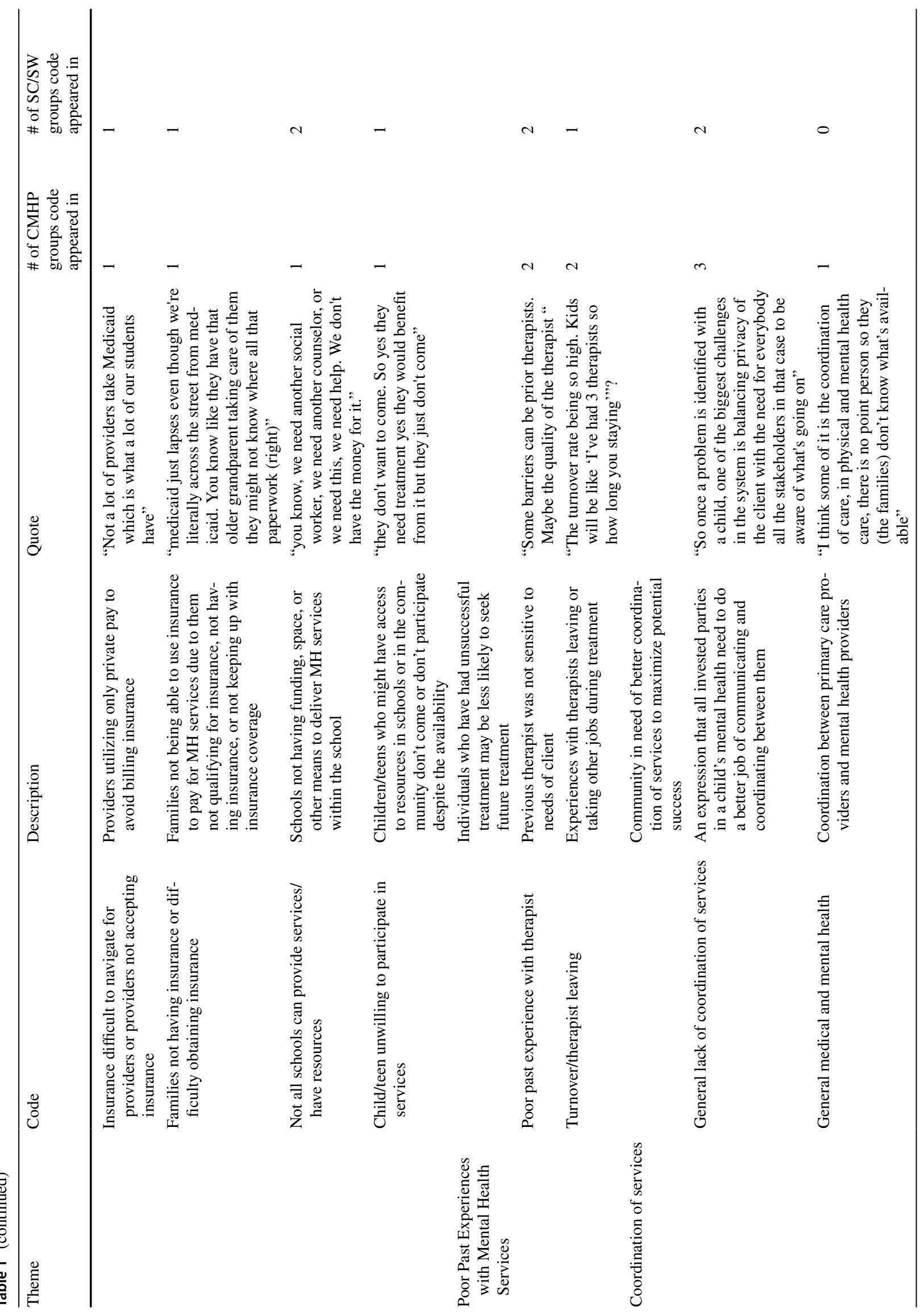




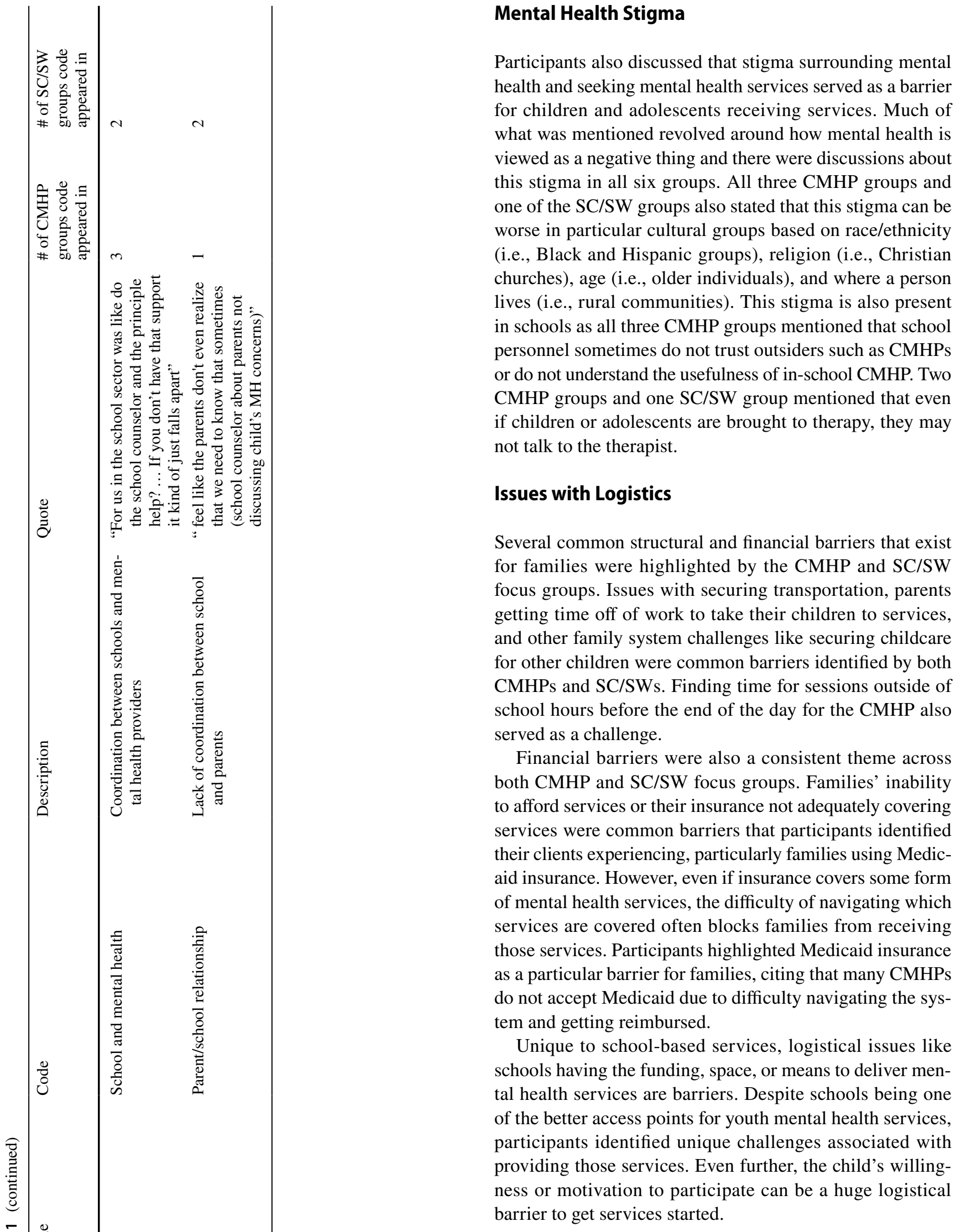

Several common structural and financial barriers that exist focus groups. Issues with securing transportation, parents getting time off of work to take their children to services, other family system challenges like securing childcare for other children were conmon barriers identified by both school hours before the end of the day for the CMHP also erved as a challenge.

Financial barriers were also a consistent theme across th CMHP and SC/SW focus groups. Families' inability afford services or their insurance not adequately covering ervices were common barriers that participants identified eir clients experiencing, particularly families using Medicinsurance. However, even if insurance covers some form of mental health services, the difficulty of navigating which ervices are covered often blocks families from receiving hose services. Participants highlighted Medicaid insurance s a particular barrier for families, citing that many CMHPs do not accept Medicaid due to difficulty navigating the sys$m$ and getting reimbursed.

Unique to school-based services, logistical issues like chools having the funding, space, or means to deliver menal health services are barriers. Despite schools being one of the better access points for youth mental health services, participants identified unique challenges associated with providing those services. Even further, the child's willingbarrier to get services started. 


\section{Poor Experiences with Mental Health}

Participants across both CMHP and SC/SW focus groups identified that poor experiences with mental health services is an important barrier to effective treatment. In particular, past experiences with mental health treatment seem to shape, and sometimes diminish, the willingness of children and their families to seek out future services. Poor therapist-child match, inadequate therapy selection, and minimal progress were cited as common reasons for poor past experiences. Finding the appropriate match for therapist and therapy type is an important barrier to overcome to improve youths' experiences. Once children are actually receiving mental health treatment, both CMHPs and SC/SWs identified that frequent therapist turnover, due to factors such as large caseloads, therapist burnout, and low wages, further contributes to poor mental health experiences and blocks adequate treatment.

\section{Poor Coordination of Services}

Given the difficulty that families experience trying to navigate the mental health care system, both CMHPs and SC/ SWs agree that the lack of coordination of services across mental health, medical, and school contexts serves as a barrier. Several CMHPs expressed their concerns about mental health treatment being a separate silo from all other services and not being able to communicate with their clients' other care providers. Furthermore, participants identified that increasing coordination and communication across contexts could provide valuable information and maximize potential success of mental health treatment. Across both CMHP and $\mathrm{SC} / \mathrm{SW}$ focus groups, participants identified the importance of bridging communication about services between mental health providers and general medical providers, schools, and the family to ensure that all parties are on the same page about how best to treat the youth.

\section{Discussion}

CMHP and SC/SW groups identified six overall themes that they believe were barriers to children and adolescents receiving adequate mental healthcare. Overall, both CMHPs and SC/ SWs were more similar than different in their responses indicating agreement about these barriers youth face. Each group identified a lack of available resources in the community and school; logistical issues at the family, school, and community levels that prevent individuals from getting services; and a lack of coordination between all relevant parties that would promote quality care. Additionally, each group identified that children and adolescents as well as relevant adults (i.e., parents and teachers) may lack the knowledge about mental health and mental health services, may have a negative view of mental health and CMHPs, or may have had a previous negative experience with CMHPs or SC/SWs. It is important to note that while these barriers fit into specific themes, they often do not occur independently of each other. In describing their experiences, CMHPs and SC/SWs often described multiple barriers interacting together to ultimately prevent youth from accessing mental health services. For example, one CMHP identified that family issues, such as parents not being able to take off work, find transportation, and arrange childcare for other children, often coincided with a lack of available providers who had sessions outside of typical school hours.

Many of the themes identified are similar to barriers identified in other studies and coincide with Reardon et al.'s (2017) four themes of systematic and structural issues, views and attitudes towards treatment, knowledge of mental health and the help-seeking process, and specific family circumstances. This not only serves to provide evidence to the existence of these barriers, but also acknowledges the relative similarity between provider perspectives and perspectives of non-providers on this topic. This may indicate that a difference in understanding of what problems are is not a sufficient explanation for why these barriers exist. However, the results of the current study diverge from previous studies in that SC/SW and CMHP also included the importance of coordination between important parties and included unique subthemes from individuals familiar with the school and community mental health environments.

Differences between the CMHP and SC/SW groups were often based on environment (i.e., CMHPs being more aware of problems unique to therapists in community and SC/SW being more aware of barriers unique to the school system). However, given the growing use of schools as a setting for youth mental health treatment, both SC/SWs and CMHPs highlighted multiple barriers specific to providing quality services within the school system, which indicates a high level of agreement on these barriers. SC/SWs work within the school system and observe many barriers firsthand, such as teachers not having adequate training to identify mental health problems in their students, limited resources to provide effective school-based therapy (i.e., not having room space or clinical training), and administrations' negative attitudes about allowing CMHPs to work with students in the school. However, CMHPs, some of whom provide services within the schools, were able to also highlight these same barriers that seem centralized to the school context.

These barriers may be best conceptualized in terms of their location in the ecological context and the point at which they exert influence, from identification to treatment. For example, lack of knowledge may influence whether mental health issues are noticed, while coordination of services would prevent quality mental healthcare after a family has sought help. These barriers are also located in one or more of the systems that the child or adolescent is located such as 
their family, school, community, and larger cultural systems. Understanding the location of these in the child's system, and when they exert influence, can help providers to personalize interventions (e.g., offering more psychoeducation to the family, or augmenting in-person with remote services) to remediate identified barriers within different systems.

\section{Clinical Implications}

To facilitate implementation of EBIs in communities and schools, barriers to implementation must be identified and minimized. In order for a child or adolescent to receive quality mental health care, each of these barriers must be avoided, successfully navigated, or overcome through other means. Attempts to make mental health services more accessible must consider ways to help families better navigate the barriers at each timepoint in the treatment process and across the different levels of the child's system. The CFIR (Damschroder et al., 2009) denotes five major domains of factors that jointly affect service implementation. Herein, we place the barriers identified in this study in the context of the CFIR domains: outer setting, inner setting (school \& CMHP entities), and process of implementation (see Fig. 1; intervention of characteristics and characteristics of individuals involved omitted as the results do not reflect a specific intervention). This framework provides a pragmatic rubric for considering the complexity and interacting nature of the identified barriers.

\section{Outer Setting}

The outer setting refers to the contextual factors outside the entity that is implementing the intervention. Both schoolbased and community providers in a given area share similar outer setting factors, such as the lack of resources (e.g., funding for providers or insurance coverages, services that meet youths' needs), which are often allocated by state or local governments and school districts, and are hard to change by individual schools or community providers. For example, SC/SW in the current study acknowledged that they often have too many students and duties to accomplish their goals; this barrier ultimately stems from the outer setting and limited funding allocated by the state government and local school districts to pay additional SC/SWs. Families of youth in need of mental health services, and their larger communities, are also couched in the outer setting; thus, lack of knowledge and stigma that prevent youth and their caregivers from seeking help are barriers to access in this setting. Implementation research on programs aimed at increasing knowledge of mental health and reducing stigma in families (Hurley et al., 2018) and youth (Milin et al., 2016) demonstrates the utility of potential mental health literacy programs to combat these outer setting barriers and may be a viable approach for communities and schools. Given outer setting stigma was identified as a prominent barrier in specific communities (e.g., religious and rural communities), identifying key trusted leaders in those communities to assist in mental health awareness may be crucial to overcome community-wide stigma. Logistical challenges experienced by youth and their families, such as additional patient needs that interfere with mental health treatment (e.g., lack of transportation or childcare) or external policies related to paying for treatment (e.g., insurance), are also couched in the outer setting. Given that schools are the primary service system for most youth, it seems these results could inform how to allocate limited resources toward improving mental healthcare in schools.

\section{School Inner Setting}

Inner setting refers to the organizational characteristics of whatever entity is implementing the intervention. In this study, the inner setting would be schools or mental healthcare organizations. One important school-based inner setting barrier in the current study was teachers' and administrators' limited knowledge of mental health, which may impact their helping behaviors or likelihood of making referrals for treatment. Additionally, the school's culture and attitudes about mental health, particularly those of teachers and administration who may be involved in deciding which students need screening and services or allocating time to mental health programming, may also be directly related to barriers like stigma and lack of resources that impact availability and effectiveness of services. Research suggests that brief interventions to improve school personnel's mental health literacy can have a direct impact on stigma and later helping behavior with students in need (Parker et al., in press; Wei et al., 2020; Whitley et al., 2018). Additionally, providing education about the reciprocal relationship between academics and mental health, as well as the financial burden of not addressing students' socioemotional needs, to administrative leadership may also be a necessary step to overcome inner setting implementation barriers like limited knowledge, stigma, and the lack of resources allocated toward mental health (Chisholm et al., 2016; Kern et al., 2017). Thus, promoting supportive and proactive attitudes, particularly about the school's role in students' mental health, may have several positive effects on inner and outer setting barriers.

\section{Community Providers Inner Setting}

Community providers may have different influences within their inner setting that are associated with various barriers. Structural characteristics of community-based settings may make it difficult to meet patients' logistical needs. For example, if CMHPs are unable to provide sufficient availability 


\section{Barriers to Implementation Identified by Providers}

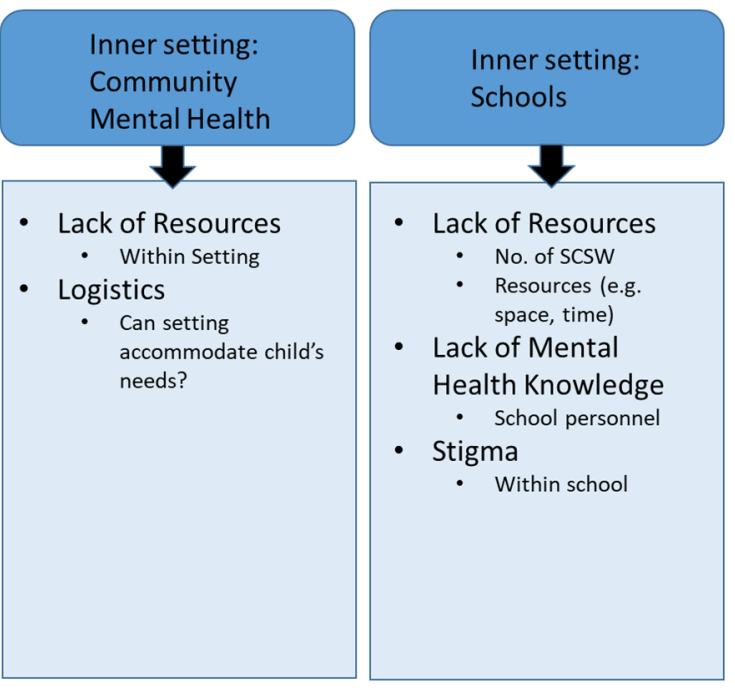

Fig. 1 Barriers to youth mental health treatment

outside of traditional school hours or take certain public insurance (e.g., Medicaid), then the clients that they can serve are greatly limited. Structural characteristics may also make it difficult to accommodate certain patients (e.g., emergency services, specialty services, or services for individuals who do not speak English) if they do not have employees or the funds and other resources necessary. The restrictions on in-person services during the COVID-19 pandemic and subsequent use of alternative intervention delivery models (e.g., greater reliance on telehealth) may have prompted a sustained shift in practice, which might solve many of the structural and logistical barriers to families seeking communitybased mental healthcare (Zhou et al., 2020); Additionally, many of the barriers identified for CMHP revolve around working with other entities and implementing larger systemic changes to the outer setting; this suggests the need for inner settings to support therapists' attempts to collaborate with other parties in a child's treatment, engage in efforts improve knowledge or reduce stigma in the community, or lobby governments to work towards policy change.

\section{Process}

Process refers to the specific interventions chosen and how an intervention is carried out. While the current study is not addressing a particular intervention, there were a number of factors identified in the mental healthcare process that impact youth receiving and completing mental health treatment. For both schools and CMHP, clients' poor experiences in therapy due to therapist or intervention fit impact the willingness to complete said intervention or even seek out
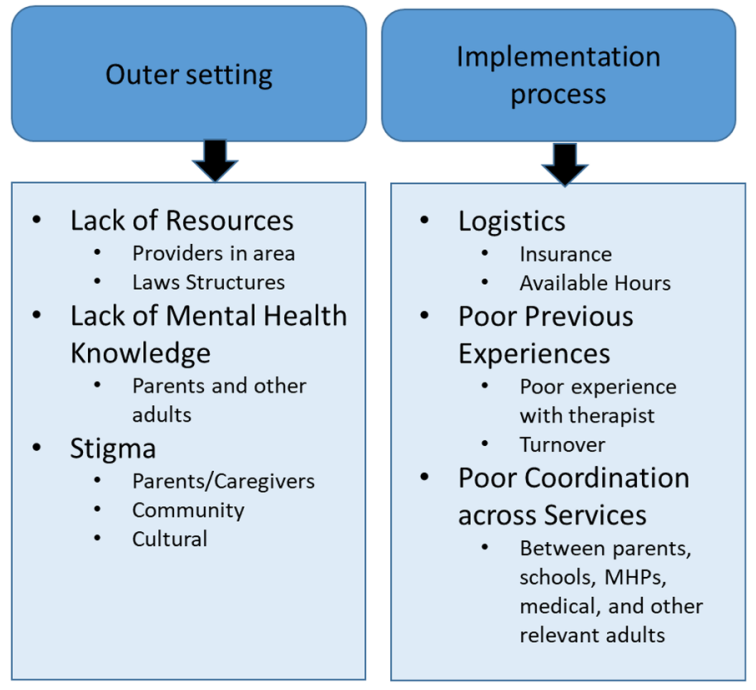

future mental health services. A key barrier was the limited collaboration and communication between different entities, such as the school, medical doctors, parents, and therapist. Increased communication about children's needs, existing services, and progress is needed to increase transparency between systems and maximize the benefit of mental health interventions for youth. For example, research suggests that a variety of strategies have been helpful to engage parents throughout the treatment process (Gross et al., 2018) and promote multisystem collaboration (Weist et al., 2012). While specific intervention choices in schools and community settings were not the focus of the current study, research suggests that selection of evidence-based programming and the use of tiered intervention, including prevention programs that encourage the socioemotional wellbeing of all students, may be key strategies to improve the process of implementation and overall effectiveness of addressing youths' wellbeing and mental health needs (Barry et al., 2013; Kern et al., 2017). Additionally, the "fit" of specific interventions, considering the research support for that intervention, the setting of delivery (e.g., school versus community), and the recipients, is a necessary consideration to tackle some of the aforementioned barriers related to poor experiences with mental health (Lyon \& Bruns, 2019).

\section{Limitations}

As with many qualitative studies, generalizability is sacrificed for the breadth of data and therefore these results may not be applicable to all situations. A different configuration of CMHPs and SC/SWs based on age, race, training, 
location, and other factors may yield different results. It should also be noted that the racial breakdown of CMHPs and $\mathrm{SC} / \mathrm{SWs}$ in this sample was significantly different and this racial discrepancy also exists between the school district and the city overall. Each of these differences could influence how the data from each group is interpreted. Additionally, while the perspective of CMHPs and SC/SWs are important, in many cases they are still expressing what they perceive to be the thoughts and beliefs of other relevant individuals (e.g., parents or teachers) which could be biased based on education, ethnocentrism, and amount of community involvement. Although focus groups are beneficial for analysis in that they allow for themes to emerge through conversation that may not be present in other methodologies such as interviews or written responses, focus groups may also pose a limitation if specific issues arise, such as one individual's perspective dominating conversation, individuals being influenced by others, and individuals choosing not to go into depth in order to allow others to share their perspective. Furthermore, the use of grounded theory allowed researchers the ability to thoroughly describe the perspectives of CMHPs and SC/SWs using their language; however, this analysis is subject to the biases of the researchers.

\section{Conclusion}

A number of barriers exist that still make it difficult for children and adolescents to receive mental health services. While some of these barriers could potentially be addressed by individual CMHPs and SC/SWs (e.g., coordinating care between interested parties, improving knowledge, reducing stigma), it is important to note that many of the potential interventions would be activities outside of their scope of practice, need to occur outside work hours, and/or may need to be done without payment. Therefore, it becomes especially important for CMHPs, community stakeholders, school administration, and other groups to work together to best address these barriers through coordinated systemic changes. These interventions can target the family microsystem in improving knowledge and reducing stigma. Targeting schools would also be important as they are considered one of the best access points for mental health services for youth and help to reduce the disparities in access for youth from communities of color and low socioeconomic status (Ali et al., 2019). Though more difficult, these groups can also target the outer settings through lobbying school boards, government officials, and insurance companies or attempting to address mental health stigma and lack of mental health literacy through larger scale efforts. Future research should focus on determining the optimal timing for intervention, what systems to target to best address implementation barriers, and feasibility and effectiveness of different interventions.

\section{Declarations}

Conflict of interest The authors certify that they have no financial or non-financial conflict of interest and they collected data in accordance with ethical standards for research with human subjects.

Informed consent This included each participant receiving and signing an Informed Consent that detailed the study procedure, potential risks and benefits, and the rights of the participant.

\section{References}

Ali, M., West, K., Teich, J., Lynch, S., Mutter, R., \& Dubenitz, J. (2018). Utilizing of mental health services in educational setting by adolescents in the United States. Journal of School Health, 89, 393-401. https://doi.org/10.1111/josh.12753

Baggish, R., \& Hardcastle, S. (2005). Schools and mental health: An evolving partnership. In Connecticut Medicine 69(9), 535-539

Barry, M. M., Clarke, A. M., Jenkins, R., \& Patel, V. (2013). A systematic review of the effectiveness of mental health promotion interventions for young people in low and middle income countries. BMC Public Health, 13(1), 1-19.

Cappella, E., Frazier, S. L., Atkins, M. S., Schoenwald, S. K., \& Glisson, C. (2008). Enhancing schools' capacity to support children in poverty: An ecological model of school-based mental health services. Administration and Policy in Mental Health and Mental Health Services Research, 35(5), 395. https://doi.org/10.1007/ s10488-008-0182-y

Chisholm, K., Patterson, P., Torgerson, C., Turner, E., Jenkinson, D., \& Birchwood, M. (2016). Impact of contact on adolescents' mental health literacy and stigma: the SchoolSpace cluster randomised controlled trial. British Medical Journal Open, 6(2), e009435.

Corbin, J., \& Strauss, A. (2008). Basics of qualitative research. Sage Publications.

Corrigan, P. W., Druss, B. G., \& Perlick, D. A. (2014). The impact of mental illness stigma on seeking and participating in mental health care. Psychological Science in the Public Interest, Supplement, 15(2), 37-70. https://doi.org/10.1177/1529100614531398

Damschroder, L. J., Aron, D. C., Keith, R. E., Kirsh, S. R., Alexander, J. A., \& Lowery, J. C. (2009). Fostering implementation of health services research findings into practice : A consolidated framework for advancing implementation science. Implementation Science, 15, 1-15. https://doi.org/10.1186/1748-5908-4-50

Das, J., Salam, R. A., Lassi, Z., Naveed, M., Mahmood, W., Patel, V., \& Bhutta, A. (2016). Interventions for adolescent mental health: An overview of systematic reviews. Journal of Adolescent Health. https://doi.org/10.1016/j.jadohealth.2016.06.020

Dempster, R., Wildman, B., \& Keating, A. (2013). The role of stigma in parental help-seeking for child behavior problems. Journal of Clinical Child \& Adolescent Psychology, 42(1), 56-67. https:// doi.org/10.1080/15374416.2012.700504

Eccles, M. P., \& Mittman, B. S. (2006). Welcome to implementation science. Implementation Science, 1(1). https://doi.org/10.1186/ 1748-5908-1-1

Farmer, E. M. Z., Burns, B. J., Phillips, S. D., Angold, A., \& Costello, E. J. (2003). Pathways into and through mental health services 
for children and adolescents. Psychiatric Services, 54(1), 60-66. https://doi.org/10.1176/appi.ps.54.1.60

Girio-Herrera, E., Owens, J. S., \& Langberg, J. M. (2013). Perceived barriers to help-seeking among parents of at-risk Kindergarteners in rural communities. Journal of Clinical Child \& Adolescent Psychology, 42(1), 68-77. https://doi.org/10.1080/15374416. 2012.715365

Gottfredson, D. C., \& Gottfredson, G. D. (2002). Quality of schoolbased prevention programs: Results from a national survey. Journal of Research in Crime and Delinquency, 39(1), 3-35. https:// doi.org/10.1177/002242780203900101

Green, J. G., McLaughlin, K. A., Alegría, M., Costello, E. J., Gruber, M. J., Hoagwood, K., Leaf, P. J., Olin, S., Sampson, N. A., \& Kessler, R. C. (2013). School mental health resources and adolescent mental health service use. Journal of the American Academy of Child and Adolescent Psychiatry, 52(5), 501-510. https://doi.org/10.1016/j.jaac.2013.03.002

Gross, D., Belcher, H. M., Budhathoki, C., Ofonedu, M. E., \& Uveges, M. K. (2018). Does parent training format affect treatment engagement? A randomized study of families at social risk. Journal of Child and Family Studies, 27(5), 1579-1593.

Gulliver, A., Griffiths, K. M., \& Christensen, H. (2010). Perceived barriers and facilitators to mental health help-seeking in young people: A systematic review. BMC Psychiatry, 10(1), 1-9. https://doi.org/10.1186/1471-244X-10-113

Hurley, D., Allen, M. S., Swann, C., Okely, A. D., \& Vella, S. A. (2018). The development, pilot, and process evaluation of a parent mental health literacy intervention through community sports clubs. Journal of Child and Family Studies, 27(7), 2149-2160.

Jensen, E. J., Wieling, E., \& Mendenhall, T. (2020). A phenomenological study of clinicians' perspectives on barriers to rural mental health care. Journal of Rural Mental Health, 44(1), 51-61. https:// doi.org/10.1037/rmh0000125

Kern, L., Mathur, S. R., Albrecht, S. F., Poland, S., Rozalski, M., \& Skiba, R. J. (2017). The need for school-based mental health services and recommendations for implementation. School Mental Health, 9(3), 205-217.

Kim, H. W., \& Salyers, M. P. (2008). Attitudes and perceived barriers to working with families of persons with severe mental illness: Mental health professionals' perspectives. Community Mental Health Journal, 44(5), 337-345. https://doi.org/10.1007/ s10597-008-9135-x

Kirk, M. A., Kelley, C., Yankey, N., Birken, S. A., Abadie, B., \& Damschroder, L. (2016). A systematic review of the use of the consolidated framework for implementation research. Implementation Science, 11(1). https://doi.org/10.1186/s13012-016-0437-z

Lyon, A. R., \& Bruns, E. J. (2019). From evidence to impact: Joining our best school mental health practices with our best implementation strategies. School Mental Health, 11(1), 106-114.

Merikangas, K. R., He, J. P., Burstein, M., Swendsen, J., Avenevoli, S., Case, B., Georgiades, K., Heaton, L., Swanson, S., \& Olfson, M. (2011). Service utilization for lifetime mental disorders in U.S. adolescents: Results of the national comorbidity survey. Journal of the American Academy of Child and Adolescent Psychiatry. https://doi.org/10.1016/j.jaac.2010.10.006

Milin, R., Kutcher, S., Lewis, S. P., Walker, S., Wei, Y., Ferrill, N., \& Armstrong, M. A. (2016). Impact of a mental health curriculum on knowledge and stigma among high school students: A randomized controlled trial. Journal of the American Academy of Child \& Adolescent Psychiatry, 55(5), 383-391.
Parker, B. L., Anderson, M., Batterham, P. J., Gayed, A., SuboticKerry, M., Achilles, M. R., ... \& O’Dea, B. (in press). Building Educators' Skills in Adolescent Mental Health (BEAM): Examining the acceptability and preliminary effectiveness of a web-based training program for Australian secondary school teachers. JMIR Mental Health, 1-29.

Pullmann, M. D., Vanhooser, S., Hoffman, C., \& Anne, C. (2010). Barriers to and supports of family participation in a rural system of care for children with serious emotional problems. Community Mental Health Journal, 46, 211-220. https://doi.org/10.1007/ s10597-009-9208-5

Reardon, T., Harvey, K., Baranowska, M., Brien, D. O., Smith, L., \& Creswell, C. (2017). What do parents perceive are the barriers and facilitators to accessing psychological treatment for mental health problems in children and adolescents? A systematic review of qualitative and quantitative studies. European Child \& Adolescent Psychiatry, 26(6), 623-647. https://doi.org/10.1007/ s00787-016-0930-6

Repie, M. S. (2005). A school mental health issues survey from the perspective of regular and special education teachers, school counselors, and school psychologists. Education and Treatment of Children, 28(3), 279-298.

Splett, J. W., Fowler, J., Weist, M. D., McDaniel, H., \& Dvorsky, M. (2013). The critical role of school psychology in the school mental health movement. Psychology in the Schools, 50(3), 245-258. https://doi.org/10.1002/pits.21677

Stevens, J., Kelleher, K. J., Ward-Estes, J., \& Hayes, J. (2006). Perceived barriers to treatment and psychotherapy attendance in child community mental health centers. Community Mental Health Journal, 42(5), 449-458. https://doi.org/10.1007/ s10597-006-9048-5

Wei, Y., Carr, W., Alaffe, R., \& Kutcher, S. (2020). Mental health literacy development: Application of online and in-person professional development for preservice teachers to address knowledge, stigma, and help-seeking intentions. Canadian Journal of Behavioural Science/revue Canadienne Des Sciences Du Comportement, 52(2), 107.

Weist, M. D., Mellin, E. A., Chambers, K. L., Lever, N. A., Haber, D., \& Blaber, C. (2012). Challenges to collaboration in school mental health and strategies for overcoming them. Journal of School Health, 82(2), 97-105.

Weist, M. D., Myers, P., Danforth, J., McNeil, D. W., Ollendick, T. H., \& Hawkins, R. (2000). Expanded school mental health services: Assessing needs related to school level and geography. Сотmunity Mental Health Journal, 36(3), 259-273.

Whitley, J., Smith, J. D., Vaillancourt, T., \& Neufeld, J. (2018). Promoting mental health literacy among educators: A critical aspect of school-based prevention and intervention. Handbook of schoolbased mental health promotion, 143-165.

Zhou, X., Snoswell, C. L., Harding, L. E., Bambling, M., Edirippulige, S., Bai, X., \& Smith, A. C. (2020). The role of telehealth in reducing the mental health burden from COVID-19. Telemedicine and e-Health, 26(4), 377-379.

Publisher's Note Springer Nature remains neutral with regard to jurisdictional claims in published maps and institutional affiliations. 